\title{
Pacific
}

Journal of

Mathematics

\section{ON ZERO-DIVISOR GRAPHS OF BOOLEAN RINGS}

\author{
Ali Mohammadian
}




\title{
ON ZERO-DIVISOR GRAPHS OF BOOLEAN RINGS
}

\author{
ALI MOHAMMADIAN
}

\begin{abstract}
The zero-divisor graph of a ring $R$ is the graph whose vertices consist of the nonzero zero-divisors of $\boldsymbol{R}$ in which two distinct vertices $\boldsymbol{a}$ and $\boldsymbol{b}$ are adjacent if and only if either $a b=0$ or $b a=0$. In this paper, we investigate some properties of zero-divisor graphs of Boolean rings. Among other results, we prove that for any two rings $R$ and $S$ with $\Gamma(R) \simeq \Gamma(S)$, if $R$ is Boolean and $|R|>4$, then $R \simeq S$.
\end{abstract}

\section{Introduction}

Throughout the paper, $R$ denotes a ring, not necessarily with identity, and $\mathcal{D}(R)$ denotes the set of all zero-divisors of $R$. If $X$ is either an element or a subset of $R$, then the left annihilator of $X$ is $\operatorname{Ann}_{\ell}(X)=\{a \in R \mid a X=0\}$ and the right annihilator of $X$, denoted by $\operatorname{Ann}_{r}(X)$, is similarly defined. For any subset $Y$ of $R$, we let $Y^{*}=Y \backslash\{0\}$. The zero-divisor graph of $R$, denoted by $\Gamma(R)$, is a graph with the vertex set $\mathcal{D}(R)^{*}$ such that two vertices $x$ and $y$ are joined by an undirected edge if and only if $x \neq y$ and either $x y=0$ or $y x=0$. Notice that a ring $R$ is a domain if and only if $\Gamma(R)$ is the null graph. For a commutative ring $R$ with identity, the definition of a zero-divisor graph of $R$ that was first introduced in [Beck 1988] coincides with the above definition of $\Gamma(R)$. The zero-divisor graph concept for noncommutative rings was first defined in [Redmond 2002]. The zero-divisor graphs offer a graphical representation of rings so that we may discover some new algebraic properties of rings that are hidden from the viewpoint of classical ring theorists. For an instance, using the notion of a zero-divisor graph, it has been proven in [Redmond 2004] that for any finite ring $R, \sum_{x \in R}\left|\operatorname{Ann}_{\ell}(x) \backslash \operatorname{Ann}_{r}(x)\right|$ is even. A simple proof of this result is given in [Akbari and Mohammadian 2007].

Let us recall some definitions regarding graph theory and ring theory. For a vertex $v$ of a graph $G, \mathcal{N}(v)$ denotes the set of all vertices of $G$ adjacent to $v$, and the degree of $v$ is defined by $|\mathcal{N}(v)|$. A graph $G$ is called a star if $G$ contains at least two vertices and there exists a vertex that is joined to all other vertices

This research was in part supported by a grant from IPM.

MSC2000: 05C25, 06E20, 16P10.

Keywords: Boolean ring, reduced ring, zero-divisor graph. 
and $G$ has no other edges. A path $\mathcal{P}$ in a graph $G$ is a sequence of distinct vertices $v_{1}, v_{2}, \ldots, v_{k+1}$ in which every two consecutive vertices are adjacent. The number $k$ is called the length of $\mathcal{P}$. For two vertices $u$ and $v$ in a graph $G$, the distance between $u$ and $v$, denoted by $d(u, v)$, is the length of the shortest path between $u$ and $v$, if such a path exists; otherwise, we define $d(u, v)=\infty$. The diameter of a graph $G$ is defined by $\operatorname{diam} G=\sup \{d(u, v) \mid u$ and $v$ are distinct vertices of $G\}$. In [Redmond 2002] it was shown that for any $\operatorname{ring} R$, diam $\Gamma(R) \leqslant 3$. Furthermore, two graphs $G_{1}$ and $G_{2}$ are said to be isomorphic if there is a bijective map $\varphi$ between the vertex set of $G_{1}$ and the vertex set of $G_{2}$ such that the adjacency relation is preserved. Finally, we recall that a ring is called reduced if it has no nonzero nilpotent elements. A ring whose elements are all idempotent is called Boolean. We denote by $\mathbb{Z}_{n}$ the ring of integers modulo $n$ and by $\mathbb{F}_{q}$ the field with $q$ elements.

In this article we study the zero-divisor graphs of Boolean rings. We show that for any reduced ring $R$ that is not a domain, $\Gamma(R)$ is isomorphic to the zero-divisor graph of a nonreduced ring, provided that $\Gamma(R)$ is a star. As a consequence, we prove that Boolean rings with more than four elements are determined by their zero-divisor graphs.

\section{The results}

In [Akbari and Mohammadian 2006, Theorem 17], it is proven that for any finite ring $R$ that is not a field, if $\Gamma(R)$ is isomorphic to the zero-divisor graph of a reduced ring $S$, then $R \simeq S$, unless $S \simeq \mathbb{Z}_{2} \times \mathbb{F}_{q}$, where either $q=2$ or $(q+1) / 2$ is a prime power. Since for any finite field $F, \Gamma\left(\mathbb{Z}_{2} \times F\right)$ is a star, the following theorem presents an analogue of this result for the general case.

Remark 1. Let $\left\{\mathcal{A}_{i}\right\}_{i \in I}$ and $\left\{\mathcal{B}_{j}\right\}_{j \in J}$ be two families of commutative domains with identity, where $|I| \geqslant 2$. In [Anderson et al. 2003, Theorem 2.1], it is shown that $\Gamma\left(\prod_{i \in I} \mathcal{A}_{i}\right) \simeq \Gamma\left(\prod_{j \in J} \mathcal{B}_{j}\right)$ if and only if there is a bijective map $\pi: I \rightarrow J$ such that $\left|\mathcal{A}_{i}\right|=\left|\mathcal{B}_{\pi(i)}\right|$ for all $i \in I$. Hence there are many examples of nonisomorphic pairs of infinite reduced commutative rings whose zero-divisor graphs are isomorphic.

Theorem 2. Let $S$ be a reduced ring such that $S$ is not a domain and $\Gamma(S)$ is not a star. If $R$ is a ring such that $\Gamma(R) \simeq \Gamma(S)$, then $R$ is also a reduced ring.

Proof. We recall a well-known fact about reduced rings: for all elements $x$ and $y$ of a reduced ring $T, x y=0$ if and only if $y x=0$. For this, note that if $x y=0$ for some elements $x, y \in T$, then $(y x)^{2}=0$ and since $T$ is reduced, we find that $y x=0$. This fact implies that if two vertices $u$ and $v$ of $\Gamma(S)$ are adjacent, then $u v=v u=0$. We use this property frequently in what follows. We also state two properties of $\Gamma(S)$ : 
(i) For every two adjacent vertices $u$ and $v$ of $\Gamma(S)$ with at least one common neighbor, $u+v$ is a vertex of $\Gamma(S)$ and $\mathcal{N}(u+v)=\mathcal{N}(u) \cap \mathcal{N}(v)$. For this, note that if $x \in \mathcal{N}(u) \cap \mathcal{N}(v)$, then $x u=x v=0$, and hence $x(u+v)=0$. Also, $u+v \neq 0$ since $u v=0$ and $S$ is reduced. Therefore, $x \in \mathcal{N}(u+v)$. Conversely, if $x \in \mathcal{N}(u+v)$, then $(x u) u=x(u+v) u=0$ and thus $u(x u)=0$. Therefore $(x u)^{2}=0$ and so $x u=0$. This means that $x \in \mathcal{N}(u)$ and with a similar argument, we find that $x \in \mathcal{N}(v)$, as required.

(ii) For every three mutually adjacent vertices $u, v$ and $w$ of $\Gamma(S)$, we have $\mathcal{N}(u) \nsubseteq$ $\mathcal{N}(v) \cup \mathcal{N}(w)$. Indeed, it easily seen that $v+w \in \mathcal{N}(u) \backslash(\mathcal{N}(v) \cup \mathcal{N}(w))$.

Suppose that $R$ is a ring with $\Gamma(R) \simeq \Gamma(S)$. So properties (i) and (ii) also hold for $\Gamma(R)$. To the contrary, assume that $a^{2}=0$ for some element $a \in R^{*}$.

Since $S$ is reduced, [Akbari and Mohammadian 2006, Corollary 4] yields that $\Gamma(R)$ has at least two vertices. Note that $a$ is not adjacent to all other vertices of $\Gamma(R)$. To prove this, suppose otherwise. Since $\Gamma(R)$ is not a star, there exist two adjacent vertices $x, y \in \mathcal{N}(a)$. So $\mathcal{N}(x) \subseteq \mathcal{N}(a) \cup \mathcal{N}(y)$, which contradicts (ii). Moreover, we have $|\mathcal{N}(a)| \geqslant 2$. For this, suppose otherwise. Since $\Gamma(R)$ is a connected graph [Redmond 2002] with at least two vertices, we may assume that $\mathcal{N}(a)=\{b\}$ for some vertex $b$ of $\Gamma(R)$. From $a+b \in \operatorname{Ann}_{\ell}(a) \cup \operatorname{Ann}_{r}(a)$, we conclude that $a+b=0$. Hence $b=-a$ and therefore $\Gamma(R)$ is a star on two vertices, a contradiction.

We claim that either $R a=\{0, a\}$ or $a R=\{0, a\}$. Suppose that there exist two elements $b \in R a \backslash\{0, a\}$ and $c \in a R \backslash\{0, a\}$. If $b \neq c$, then $a, b$ and $c$ are three mutually adjacent vertices and $\mathcal{N}(a) \subseteq \mathcal{N}(b) \cup \mathcal{N}(c)$, which contradicts (ii). Hence $b=c$. For some vertex $d \in \mathcal{N}(a) \backslash\{b\}$, the vertices $a, b$ and $d$ are mutually adjacent and $\mathcal{N}(a) \subseteq \mathcal{N}(b) \cup \mathcal{N}(d)$, which again contradicts (ii). Since $R a \neq\{0\}$ and $a R \neq\{0\}$, the claim is proved.

We assume that $R a=a R=\{0, a\}$. For any two vertices $x, y \notin \mathcal{N}(a)$, we have $x a=y a=a$. Thus $(x y) a=a$ and so $x y \neq 0$. This means that every edge of $\Gamma(R)$ has at least one endpoint in $\mathcal{N}(a)$. Working towards a contradiction, assume that no two vertices in $\mathcal{N}(a)$ are adjacent. This means that $\Gamma(R)$, and so $\Gamma(S)$ is a bipartite graph, and using [Akbari et al. 2003, Theorem 2.4], $\Gamma(S)$ and thus $\Gamma(R)$ is a complete bipartite graph. Let $r \notin \mathcal{N}(a)$ and $s \in \mathcal{N}(a) \cap \mathcal{N}(r)$. Since $\Gamma(R)$ is a complete bipartite graph and $a+s \in \mathcal{N}(a), r$ is adjacent to $a+s$. Therefore $a=r(a+s) r=0$, a contradiction. Hence there are two adjacent vertices $b, c \in \mathcal{N}(a)$. We now consider the two following cases.

Case I. Suppose that $a$ together with one of the elements $b, c$ are contained in one of the one-sided annihilators of the third element. Without loss of generality, assume that $\{a, c\} \in \operatorname{Ann}_{\ell}(b)$. By (i), there exists a vertex $d$ in $\Gamma(R)$ such that $d \notin\{a, b\}$ and $\mathcal{N}(d)=\mathcal{N}(a) \cap \mathcal{N}(b)$. If $b \neq a+c$, then $a+c \in \mathcal{N}(a) \cap \mathcal{N}(b)$, and 
hence $a=d(a+c) d=0$, a contradiction. Thus $b=a+c$, and it follows from $a b=0$ that $a c=0$. Moreover, if $c a \neq 0$, then $a=c a=c b-c^{2}=-c^{2}$, which contradicts $d \in \mathcal{N}(c) \backslash \mathcal{N}(a)$. Therefore $c a=0$ and so $c^{2}=c b-c a=0$. Since $b=a+c$, we find that the product of any two elements of $\{a, b, c\}$ is zero.

Suppose towards a contradiction that there is a vertex $r \in(\mathcal{N}(b) \cap \mathcal{N}(c)) \backslash\{a\}$. We have $r a r=r(b-c) r=0$ and by $R a=a R=\{0, a\}$, we deduce that $r \in \mathcal{N}(a)$. By (i), there exists a vertex $s$ in $\Gamma(R)$ such that $\mathcal{N}(s)=\mathcal{N}(a) \cap \mathcal{N}(r)$. This implies that $s a s=s(b-c) s=0$, since $\{b, c\} \subseteq \mathcal{N}(a) \cap \mathcal{N}(r)$. On the other hand, $s \notin \mathcal{N}(a)$ and $R a=a R=\{0, a\}$ yields that $s a s=a$, a contradiction. This establishes that $\mathcal{N}(b) \cap \mathcal{N}(c)=\{a\}$.

For convenience and without loss of generality, assume that $c d=0$. From $\{b, c\} \subseteq \operatorname{Ann}_{r}(c), d \notin \mathcal{N}(a) \cup \mathcal{N}(b)$ and $\mathcal{N}(b) \cap \mathcal{N}(c)=\{a\}$, we have $R c=\{0, c\}$. Therefore $\left[R: \operatorname{Ann}_{\ell}(a) \cap \operatorname{Ann}_{\ell}(c)\right] \leqslant\left[R: \operatorname{Ann}_{\ell}(a)\right]\left[R: \operatorname{Ann}_{\ell}(c)\right]=|R a||R c|=4$. Since $\mathcal{N}(b) \cap \mathcal{N}(c)=\{a\}$ and the product of any two elements of $\{a, b, c\}$ is zero, we find that $\operatorname{Ann}_{\ell}(a) \cap \operatorname{Ann}_{\ell}(c)=\{0, a, b, c\}$. This yields that $|R| \leqslant 16$. Using (i), let $e$ be a vertex of $\Gamma(R)$ in which $\mathcal{N}(e)=\mathcal{N}(a) \cap \mathcal{N}(c)$. It is not hard to see that

$$
R=\{0, a, b, c\} \cup(d+\{0, a, b, c\}) \cup(e+\{0, a, b, c\}) \cup(d+e+\{0, a, b, c\}) .
$$

Therefore $\operatorname{Ann}_{\ell}(a)=\operatorname{Ann}_{r}(a)=\{0, a, b, c\} \cup(d+e+\{0, a, b, c\})$. Because $e \notin$ $\mathcal{N}(a) \cup \mathcal{N}(c), R a=\{0, a\}$ and $R c=\{0, c\}$, we conclude that $e a=a$ and $e c=c$. Therefore $e b=b$ and by $b \in \mathcal{N}(a) \cap \mathcal{N}(c)$, we obtain that $b e=0$. Furthermore, $e \notin \mathcal{N}(a) \cup \mathcal{N}(c)$ and $\mathcal{N}(b) \cap \mathcal{N}(c)=\{a\}$ yield that $R b=\{0, b\}$. It follows from $d \notin \mathcal{N}(b)$ that $d+e \in \operatorname{Ann}_{\ell}(b)$, and so $\mathcal{N}(a) \subseteq \mathcal{N}(b) \cup \mathcal{N}(c)$, which contradicts (ii).

Case II. When Case I does not occur, by replacing $b$ with $c$ if necessary, we may assume that $a b=b c=c a=0$ and none of $b a, c b$ and $a c$ is zero. We have $\{a, b\} \in \mathrm{Ann}_{\ell}(c b)$, and so, applying the argument in the first paragraph of Case I for $c b$ and $b$ instead of $b$ and $c$, respectively, we obtain in particular that $b a=0$, which is a contradiction.

Next, with no loss of generality, assume that $a R=\{0, a\}$ and there exists an element $g \in R a \backslash\{0, a\}$. Since $a R=\{0, a\},-a=a$ and so $-g=g$. Also, from $g \in R a$ and $a R=\{0, a\}$, we easily obtain that $a g=g a=0$. By (i), there exists a vertex $h$ in $\Gamma(R)$ such that $\mathcal{N}(h)=\mathcal{N}(a) \cap \mathcal{N}(g)$. We claim that $\operatorname{Ann}_{r}(a) \subseteq$ $\operatorname{Ann}_{r}(h) \cup\{0, a, g, a+g\}$. Suppose $x \in \operatorname{Ann}_{r}(a) \backslash\left(\operatorname{Ann}_{r}(h) \cup\{a, g\}\right)$. Since $g \in R a$ and $\mathcal{N}(h)=\mathcal{N}(a) \cap \mathcal{N}(g)$, we conclude that $x \in \operatorname{Ann}_{\ell}(h)$. Moreover, $h \notin \mathcal{N}(a)$ and $a R=\{0, a\}$, so $a h=a$. We have $a+g \in \mathcal{N}(a) \cap \mathcal{N}(g)$ and $(a+g) h=a+g$, and hence $h(a+g)=0$. These equalities yield that $h(a+g+x)=h x \neq 0$ and $(a+g+x) h=a+g \neq 0$. On the other hand, $a(a+g+x)=0$, so it follows from $g \in R a$ and $\mathcal{N}(h)=\mathcal{N}(a) \cap \mathcal{N}(g)$ that $a+g+x=0$. Therefore, $x=a+g$, and the claim is proved. $\operatorname{Since}_{\mathrm{Ann}_{r}}(a), \operatorname{Ann}_{r}(h)$ and $\{0, a, g, a+g\}$ 
are three additive subgroups of $R$ in which $\operatorname{Ann}_{r}(a) \subseteq \operatorname{Ann}_{r}(h) \cup\{0, a, g, a+g\}$ and $a \in \operatorname{Ann}_{r}(a) \backslash \operatorname{Ann}_{r}(h)$, we deduce that $\operatorname{Ann}_{r}(a)=\{0, a, g, a+g\}$. Applying (ii), there exists a vertex $y \in \mathcal{N}(a) \backslash(\mathcal{N}(g) \cup \mathcal{N}(a+g))$. We have $y a=0$ and $a y \neq 0$. By $\left[R: \operatorname{Ann}_{r}(a)\right]=|a R|$, we conclude that $R=\operatorname{Ann}_{r}(a) \cup y+\operatorname{Ann}_{r}(a)$. It follows from $\operatorname{Ann}_{r}(a)=\{0, a, g, a+g\}$ that $R a=\{0\}$, a contradiction. Now the proof is complete.

Example 3. The condition on $\Gamma(S)$ in Theorem 2 is necessary. For examples involving infinite rings, let $\mathscr{S}$ be an arbitrary infinite domain, $\mathscr{R}$ be the polynomial ring in the set of variables $\{\mathrm{x}\} \cup\left\{\mathrm{x}_{\alpha} \mid \alpha \in \mathscr{Y}\right\}$ with coefficients in $\mathbb{Z}_{2}$, and $\mathscr{I}$ be the ideal of $\mathscr{R}$ generated by $\left\{\mathrm{x}^{2}\right\} \cup\left\{\mathrm{xx}_{\alpha}-\mathrm{x} \mid \alpha \in \mathscr{S}\right\} \cup\left\{\mathrm{x}_{\alpha} \mathrm{x}-\mathrm{x} \mid \alpha \in \mathscr{S}\right\}$. It is easy to verify that $\Gamma(\mathscr{R} / \mathscr{I})$ is a star on $|\mathscr{I}|$ vertices and $\mathrm{x}+\mathscr{I}$ is that vertex which is adjacent to all other vertices of the graph. Therefore $\Gamma(\mathscr{R} / \mathscr{T}) \simeq \Gamma\left(\mathbb{Z}_{2} \times \mathscr{S}\right)$, while $\mathscr{R} / \mathscr{I}$ is not reduced.

Remark 4. It is easy to establish that every reduced ring whose zero-divisor graph is a star is isomorphic to the direct product of $\mathbb{Z}_{2}$ and a domain. For this, let $R$ be a reduced ring with $\Gamma(R)$ a star and let $e$ be that vertex which is adjacent to all other vertices of $\Gamma(R)$. Obviously, $e$ is idempotent, and using the fact that all idempotent elements of a reduced ring are central, we may write $R \simeq e R \times(1-e) R$. Since $\Gamma(R)$ is a star, we clearly conclude that $e R=\{0, e\}$ and $(1-e) R$ is a domain, as required. From this, Theorem 2, Example 3, and [Akbari and Mohammadian 2006, Theorem 17], we imply that for every reduced ring $R$ that is not a domain, $\Gamma(R)$ is isomorphic to the zero-divisor graph of a nonreduced ring if and only if $\Gamma(R)$ is either an infinite star or a star with $q$ vertices, where either $q=2$ or both $q$ and $(q+1) / 2$ are prime powers.

In [LaGrange 2007, Theorem 4.1], it is shown that if $R$ and $S$ are two commutative rings with identity such that $S$ is a Boolean ring with more than four elements and $\Gamma(R) \simeq \Gamma(S)$, then $R \simeq S$. In what follows, we generalize this result to every arbitrary ring $R$. We need the following easy lemmas.

Lemma 5. Let $R$ be a ring such that all elements in $\mathcal{D}(R)$ are idempotent. Then $R$ is either a domain or a Boolean ring.

Proof. Suppose that $R$ is not a domain. By the hypotheses, $R$ is reduced. Using the fact that all idempotent elements of a reduced ring are central, $\mathcal{D}(R)$ is contained in the center of $R$. Therefore, for every two elements $a \in R$ and $z \in \mathcal{D}(R)^{*}$, we have $a z \in \mathcal{D}(R)$. Hence $(a z)^{2}=a z$, and so $\left(a^{2}-a\right) z=0$. The latter equality shows that $a^{2}-a \in \mathcal{D}(R)$ and also $\operatorname{Ann}_{\ell}\left(a^{2}-a\right)=\mathcal{D}(R)$. Thus $a^{2}-a=\left(a^{2}-a\right)^{2}=0$ for each element $a \in R$, as desired.

Lemma 6. Let $R$ be a Boolean ring with $|R|>4$. Then $\Gamma(R)$ contains no vertex adjacent to all other vertices of the graph. 
Proof by contradiction. Suppose that a vertex $r$ is adjacent to all other vertices of $\Gamma(R)$. Let $z \in \mathcal{D}(R) \backslash\{0, r\}$. We have $r(r+z)=r \neq 0$ and so $r+z$ is a nonzero-divisor idempotent of $R$. Thus $1=r+z$ is the identity of $R$ and so $R=\{0,1, r, 1-r\}$, which contradicts $|R|>4$.

Theorem 7. Let $S$ be a Boolean ring with $|S|>4$. Suppose that $R$ is a ring and $\varphi: \Gamma(R) \rightarrow \Gamma(S)$ is a graph isomorphism. Then $\varphi$ is extendable to a ring isomorphism from $R$ to $S$. In particular, $R \simeq S$.

Proof. Recall that the characteristic of every Boolean ring is 2 . We first state the following properties of $\Gamma(S)$.

(i) For every two vertices $u$ and $v$ of $\Gamma(S)$, if $\mathcal{N}(u)=\mathcal{N}(v)$, then $u=v$. For this, note that if $u \neq u v$, then $u+u v \in \mathcal{N}(v) \backslash \mathcal{N}(u)$, which is impossible. So we conclude that $u=u v$, and similarly $v=u v$, which yield that $u=v$, as desired.

(ii) For every two adjacent vertices $u$ and $v$ of $\Gamma(S)$, using (i) together with an easy argument, we find that $u+v$ is the unique vertex of $\Gamma(S)$ such that $\mathcal{N}(u+v)=\mathcal{N}(u) \cap \mathcal{N}(v)$, if $\mathcal{N}(u) \cap \mathcal{N}(v) \neq \varnothing$; and otherwise, $1=u+v$ is the identity of $S$, because in this case $u+v$ is a nonzero-divisor idempotent of $S$. Moreover, if $S$ has identity, then $v=1+u$ is the unique neighbor of $u$ in $\Gamma(S)$ such that $\mathcal{N}(u) \cap \mathcal{N}(v)=\varnothing$. For uniqueness, note that for any vertex $x \in \mathcal{N}(u)$, if $x \neq 1+u$, then $1+u+x \in \mathcal{N}(u) \cap \mathcal{N}(x)$.

(iii) For every two nonadjacent vertices $u$ and $v$ of $\Gamma(S), \mathcal{N}(u) \cup \mathcal{N}(v) \subseteq \mathcal{N}(u v)$; and if $\mathcal{N}(u) \cup \mathcal{N}(v) \subseteq \mathcal{N}(w)$ for some vertex $w$ of $\Gamma(S)$, then $\mathcal{N}(u v) \subseteq \mathcal{N}(w)$. For the second statement, let $x \in \operatorname{Ann}_{\ell}(u v)$. We have $v x \in \operatorname{Ann}_{\ell}(u)$. Since $\mathcal{N}(u) \subseteq \mathcal{N}(w), w(v x)=0$ and so $w x \in \operatorname{Ann}_{\ell}(v)$. It follows from $\mathcal{N}(v) \subseteq \mathcal{N}(w)$ that $w(w x)=0$ and thus $x \in \operatorname{Ann}_{\ell}(w)$, as required.

Since $\Gamma(R) \simeq \Gamma(S)$, the above properties also hold for $\Gamma(R)$. Using Theorem 2 and Lemma 6, $R$ is reduced. It is easily checked that $\mathcal{N}\left(z^{2}\right)=\mathcal{N}(z)$ for each vertex $z$ of $\Gamma(R)$. By (i), we have $z^{2}=z$ for every element $z \in \mathcal{D}(R)$. Applying Lemma 5 , $R$ is a Boolean ring. Define $\varphi(0)=0$. By (ii), $\Gamma(R)$ (respectively, $\Gamma(S)$ ) contains two adjacent vertices with no common neighbors if and only if $R$ (respectively, $S$ ) has identity. Since $\Gamma(R) \simeq \Gamma(S)$, either both $R$ and $S$ have identity or neither of them has identity. When the first case occurs, we define $\varphi(1)=1$. Furthermore, the properties (ii) and (iii) imply that for every two vertices $u$ and $v$ of $\Gamma(S)$, the elements

$-1+u$, if $S$ has identity;

- $u+v$, if $u$ and $v$ are adjacent and $u+v \neq 1$; and

- $u v$, if $u$ and $v$ are not adjacent, 
can be determined by $\Gamma(S)$. We claim that for every two distinct nonadjacent vertices $u$ and $v$ of $\Gamma(S)$, the element $u+v$ can also be determined by $\Gamma(S)$. First assume that $u v \notin\{u, v\}$. Using (iii), we obtain that the element $u v$ is determined by $\Gamma(S)$. By (i) and (ii), $u+u v$ is the unique vertex of $\Gamma(S)$ such that $\mathcal{N}(u)=$ $\mathcal{N}(u v) \cap \mathcal{N}(u+u v)$. This and a similar argument establish that the elements $u+u v$ and $v+u v$ are determined by $\Gamma(S)$. Since the vertices $u+u v$ and $v+u v$ are adjacent, we are done using (ii). Next, with no loss of generality, suppose that $u v=u$. In this case, the vertices $u$ and $u+v$ are adjacent, and so applying (i) and (ii), we find that $u+v$ is the unique vertex of $\Gamma(S)$ such that $\mathcal{N}(v)=\mathcal{N}(u) \cap \mathcal{N}(u+v)$. This proves the claim. Now, by $\Gamma(R) \simeq \Gamma(S)$ and the above reasonings, it is not hard to verify that $\varphi(a+b)=\varphi(a)+\varphi(b)$ and $\varphi(a b)=\varphi(a) \varphi(b)$ for all $a, b \in R$, as desired.

As an interesting fact, it is well-known that every isomorphism between multiplicative semigroups of two Boolean rings is a ring isomorphism. Obviously, Theorem 7 generalizes this fact. The following theorem asserts that the zero-divisor graph of a Boolean ring $R$ determines whether $R$ has identity or not.

Theorem 8. Let $R$ be a Boolean ring and $|R|>4$. Then $\operatorname{diam} \Gamma(R)=3$ if $R$ has identity, and otherwise diam $\Gamma(R)=2$.

Proof. We know from [Redmond 2002] that for any $\operatorname{ring} T$, diam $\Gamma(T) \leqslant 3$. First suppose that $R$ has identity. Since $|R|>4$, we can take an element $e \notin\{0,1\}$. We have $R=e R \oplus(1-e) R$, so either $|e R|>2$ or $|(1-e) R|>2$. With no loss of generality, let $f \in e R \backslash\{0, e\}$. Since $e$ and $1+e+f$ are two nonadjacent vertices with no common neighbors and $\operatorname{diam} \Gamma(R) \leqslant 3$, the result follows.

Next suppose that $R$ has no identity. Applying Lemma 6, we find diam $\Gamma(R) \geqslant 2$. Now, let $a$ and $b$ be two nonadjacent vertices of $\Gamma(R)$. Since $R$ has no identity, there exists an element $c$ such that $(a+b+a b) c \neq c$. We have $c+a c+b c+a b c \in$ $\mathcal{N}(a) \cap \mathcal{N}(b)$, which clearly completes the proof.

It is well-known that every finite Boolean ring has identity. We generalize this fact in the following theorem.

Theorem 9. Let $R$ be a Boolean ring such that $\Gamma(R)$ has a vertex of finite degree. Then $R$ has identity.

Proof. Recall that the adjoint multiplication $\circ$ of an arbitrary ring $T$ is defined by $x \circ y=x+y+x y$ for any two elements $x, y \in T$. Suppose that $a$ is a vertex of finite degree of $\Gamma(R)$ and $\mathcal{N}(a)=\left\{a_{1}, \ldots, a_{n}\right\}$ for some integer $n \geqslant 1$. Let $b=a_{1} \circ \cdots \circ a_{n}$. Clearly, $a b=0$ and $a_{i} b=a_{i}$ for all $i$. We show that $a+b$ is the identity of $R$. Indeed, it is enough to prove that $a+b$ is a nonzero-divisor. Toward a contradiction, assume that $(a+b) z=0$ for some element $z \in R^{*}$. Multiplying this equality by $a$, we find that $a z=0$, and hence $z=a_{j}$ for some $j \in\{1, \ldots, n\}$. 
Also, multiplying the equality $(a+b) z=0$ by $a_{j}$ yields that $a_{j} z=0$, which is impossible. This completes the proof.

Remark 10. The converse of Theorem 9 is not true. Let $\mathscr{R}$ be the set consisting of the empty set together with all finite unions of all left-closed right-open intervals and all left-unbounded right-open intervals of real numbers. Clearly, $\mathscr{R}$ is a Boolean ring with identity with respect to symmetric difference as the addition operation and intersection as the multiplication operation, while obviously every vertex of $\Gamma(\mathscr{R})$ has infinite degree.

We conclude the paper with the following theorem on the polynomial rings over Boolean rings.

Theorem 11. Let $R$ and $S$ be two Boolean rings such that $\Gamma(R[\mathrm{x}]) \simeq \Gamma(S[\mathrm{x}])$. Then $R \simeq S$.

Proof. Let $T$ be an arbitrary Boolean ring. $\Gamma(T[\mathrm{x}])$ is the null graph if and only if $T \simeq \mathbb{Z}_{2}$. Hence we may assume that $\mathcal{D}(R)^{*}$ and $\mathcal{D}(S)^{*}$ are both nonempty. Using Theorem 7, it suffices to establish that $\Gamma(R) \simeq \Gamma(S)$. Since finitely generated onesided ideals of von Neumann regular rings, including Boolean rings, are principal [Lam 2001, (4.23)], for each finitely generated ideal $I$ of $T$, there exists a unique element $e$ such that $I=(e)$. For a polynomial $f(\mathrm{x})=a_{n} \mathrm{x}^{n}+\cdots+a_{0} \in T[\mathrm{x}]$, let $\widehat{f(\mathrm{x})}$ be the unique element of $T$ such that $\left(a_{0}, \ldots, a_{n}\right)=(\widehat{f(\mathrm{x})})$. From [Armendariz 1974, Lemma 1], every reduced ring is Armendariz, and hence it is not hard to see that for any polynomial $f(\mathrm{x}) \in \mathcal{D}(T[\mathrm{x}])^{*}, \widehat{f(\mathrm{x})}$ is the unique element of $T$ such that $\mathcal{N}(f(\mathrm{x}))=\mathcal{N}(\widehat{f(\mathrm{x})})$.

Now, assume that $\phi: \Gamma(R[\mathrm{x}]) \rightarrow \Gamma(S[\mathrm{x}])$ is a graph isomorphism. We define $\psi: \Gamma(R) \rightarrow \Gamma(S)$ by $\psi(a)=\widehat{\phi(a)}$ for all $a \in \mathcal{D}(R)^{*}$, and we claim that $\psi$ is a graph isomorphism. If $a$ and $b$ are two adjacent vertices of $\Gamma(R)$, then $\phi(a) \in \mathcal{N}(\phi(b))=$ $\mathcal{N}(\psi(b))$. This yields that $\psi(b) \in \mathcal{N}(\phi(a))=\mathcal{N}(\psi(a))$ and therefore $\psi(a)$ and $\psi(b)$ are adjacent in $\Gamma(S)$. The converse is clearly true, and so $\psi$ preserves the adjacency relation. Moreover, if $\psi(a)=\psi(b)$ for two vertices $a$ and $b$ of $\Gamma(R)$, then $\mathcal{N}(\phi(a))=\mathcal{N}(\phi(b))$ and thus $\mathcal{N}(a)=\mathcal{N}(b)$. In particular, $\mathcal{N}(a) \cap R=\mathcal{N}(b) \cap R$. Using the property (i) of the zero-divisor graphs of Boolean rings given in the proof of Theorem 7, we deduce that $a=b$. This concludes the injectivity of $\psi$. Finally, we prove that $\psi$ is surjective. Suppose $s \in \mathcal{D}(S)^{*}$ and let

$$
r=\widehat{\phi^{-1}(s)} \text {. }
$$

Since $\mathcal{N}\left(\phi^{-1}(s)\right)=\mathcal{N}(r)$, we find that $\mathcal{N}(s)=\mathcal{N}(\phi(r))=\mathcal{N}(\psi(r))$ and hence $s=\psi(r)$. This establishes the claim and completes the proof.

Remark 12. Let $n \geqslant 2$ and $\mathscr{R}$ and $\mathscr{Y}$ be two rings which each of them is the direct product of $n$ arbitrary finite fields. Using the result mentioned in Remark 1, it is 
easily checked that $\Gamma(\mathscr{R}[\mathrm{x}]) \simeq \Gamma(\mathscr{Y}[\mathrm{x}])$. Therefore the conclusion of Theorem 11 is not true if one of $R$ and $S$ is not Boolean.

\section{References}

[Akbari and Mohammadian 2006] S. Akbari and A. Mohammadian, "Zero-divisor graphs of noncommutative rings", J. Algebra 296:2 (2006), 462-479. MR 2007a:16046 Zbl 1113.16038

[Akbari and Mohammadian 2007] S. Akbari and A. Mohammadian, "On zero-divisor graphs of finite rings", J. Algebra 314:1 (2007), 168-184. MR 2008f:16048 Zbl 1122.16015

[Akbari et al. 2003] S. Akbari, H. R. Maimani, and S. Yassemi, "When a zero-divisor graph is planar or a complete $r$-partite graph", J. Algebra 270:1 (2003), 169-180. MR 2004h:13025 Zbl 1032.13014

[Anderson et al. 2003] D. F. Anderson, R. Levy, and J. Shapiro, "Zero-divisor graphs, von Neumann regular rings, and Boolean algebras", J. Pure Appl. Algebra 180:3 (2003), 221-241. MR 2003m: 13007 Zbl 1076.13001

[Armendariz 1974] E. P. Armendariz, “A note on extensions of Baer and P.P.-rings”, J. Austral. Math. Soc. 18 (1974), 470-473. MR 51 \#3224 Zbl 0292.16009

[Beck 1988] I. Beck, “Coloring of commutative rings”, J. Algebra 116:1 (1988), 208-226. MR 89i: 13006 Zbl 0654.13001

[LaGrange 2007] J. D. LaGrange, "Complemented zero-divisor graphs and Boolean rings", J. Algebra 315:2 (2007), 600-611. MR 2008h:13012 Zbl 1133.13005

[Lam 2001] T. Y. Lam, A first course in noncommutative rings, 2nd ed., Graduate Texts in Mathematics 131, Springer, New York, 2001. MR 2002c:16001 Zbl 0980.16001

[Redmond 2002] S. P. Redmond, "The zero-divisor graph of a non-commutative ring”, pp. 39-47 in Commutative rings, edited by A. Badawi, Nova Sci. Publ., Hauppauge, NY, 2002. MR 2037657 Zbl 1195.16038

[Redmond 2004] S. P. Redmond, "Structure in the zero-divisor graph of a noncommutative ring", Houston J. Math. 30:2 (2004), 345-355. MR 2005d:16002 Zbl 1064.16033

Received June 5, 2010. Revised August 31, 2010.

\section{Ali Mohammadian}

SCHOOL OF MATHEMATICS

INSTITUTE FOR RESEARCH IN FUNDAMENTAL SCIENCES (IPM)

NIAVARAN SQUARE

P.O. BOX 19395-5746

TEHRAN

IRAN

ali_m@ipm.ir 


\title{
PACIFIC JOURNAL OF MATHEMATICS
}

\author{
http://www.pjmath.org \\ Founded in 1951 by
}

E. F. Beckenbach (1906-1982) and F. Wolf (1904-1989)

\section{EDITORS}

V. S. Varadarajan (Managing Editor)

Department of Mathematics

University of California

Los Angeles, CA 90095-1555

pacific@math.ucla.edu

Vyjayanthi Chari

Department of Mathematics

University of California

Riverside, CA 92521-0135

chari@math.ucr.edu

\section{Robert Finn}

Department of Mathematics Stanford University

Stanford, CA 94305-2125

finn@math.stanford.edu

Kefeng Liu

Department of Mathematics

University of California

Los Angeles, CA 90095-1555

liu@math.ucla.edu
Darren Long

Department of Mathematics

University of California

Santa Barbara, CA 93106-3080

long@math.ucsb.edu

Jiang-Hua Lu

Department of Mathematics

The University of Hong Kong

Pokfulam Rd., Hong Kong jhlu@maths.hku.hk

Alexander Merkurjev

Department of Mathematics University of California

Los Angeles, CA 90095-1555 merkurev@math.ucla.edu
Sorin Popa

Department of Mathematics

University of California

Los Angeles, CA 90095-1555

popa@math.ucla.edu

Jie Qing

Department of Mathematics

University of California

Santa Cruz, CA 95064

qing@ cats.ucsc.edu

Jonathan Rogawski

Department of Mathematics

University of California

Los Angeles, CA 90095-1555

jonr@math.ucla.edu

\section{PRODUCTION}

pacific@math.berkeley.edu

Silvio Levy, Scientific Editor Matthew Cargo, Senior Production Editor

ACADEMIA SINICA, TAIPEI

CALIFORNIA INST. OF TECHNOLOGY

INST. DE MATEMÁTICA PURA E APLICADA

KEIO UNIVERSITY

MATH. SCIENCES RESEARCH INSTITUTE

NEW MEXICO STATE UNIV.

OREGON STATE UNIV.

\section{SUPPORTING INSTITUTIONS}

STANFORD UNIVERSITY
UNIV. OF BRITISH COLUMBIA
UNIV. OF CALIFORNIA, BERKELEY
UNIV. OF CALIFORNIA, DAVIS
UNIV. OF CALIFORNIA, LOS ANGELES
UNIV. OF CALIFORNIA, RIVERSIDE
UNIV. OF CALIFORNIA, SAN DIEGO
UNIV. OF CALIF., SANTA BARBARA

UNIV. OF CALIF., SANTA CRUZ

UNIV. OF MONTANA

UNIV. OF OREGON

UNIV. OF SOUTHERN CALIFORNIA

UNIV. OF UTAH

UNIV. OF WASHINGTON

WASHINGTON STATE UNIVERSITY

These supporting institutions contribute to the cost of publication of this Journal, but they are not owners or publishers and have no responsibility for its contents or policies.

See inside back cover or www.pjmath.org for submission instructions.

The subscription price for 2011 is US \$420/year for the electronic version, and \$485/year for print and electronic.

Subscriptions, requests for back issues from the last three years and changes of subscribers address should be sent to Pacific Journal of Mathematics, P.O. Box 4163, Berkeley, CA 94704-0163, U.S.A. Prior back issues are obtainable from Periodicals Service Company, 11 Main Street, Germantown, NY 12526-5635. The Pacific Journal of Mathematics is indexed by Mathematical Reviews, Zentralblatt MATH, PASCAL CNRS Index, Referativnyi Zhurnal, Current Mathematical Publications and the Science Citation Index.

The Pacific Journal of Mathematics (ISSN 0030-8730) at the University of California, c/o Department of Mathematics, 969 Evans Hall, Berkeley, CA 94720-3840, is published monthly except July and August. Periodical rate postage paid at Berkeley, CA 94704, and additional mailing offices. POSTMASTER: send address changes to Pacific Journal of Mathematics, P.O. Box 4163, Berkeley, CA 94704-0163.

PJM peer review and production are managed by EditFLOW ${ }^{\mathrm{TM}}$ from Mathematical Sciences Publishers.

PUBLISHED BY PACIFIC JOURNAL OF MATHEMATICS

at the University of California, Berkeley 94720-3840

A NON-PROFIT CORPORATION

Typeset in IATEX

Copyright $(2011$ by Pacific Journal of Mathematics 


\section{PACIFIC JOURNAL OF MATHEMATICS}

Volume $251 \quad$ No. $2 \quad$ June 2011

Two Kazdan-Warner-type identities for the renormalized volume coefficients and the Gauss-Bonnet curvatures of a Riemannian metric

BIN GUO, ZHENG-CHAO HAN and HAIZHONG LI

Gonality of a general ACM curve in $\mathbb{P}^{3}$

ROBIN HARTSHORNE and ENRICO SCHLESINGER

Universal inequalities for the eigenvalues of the biharmonic operator on submanifolds

SAÏD ILIAS and OLA MAKHOUL

Multigraded Fujita approximation

SHIN-YaO Jow

Some Dirichlet problems arising from conformal geometry

QI-RUi Li and WEIMIN SHENG

Polycyclic quasiconformal mapping class subgroups

KATSUHIKO MATSUZAKI

On zero-divisor graphs of Boolean rings

ALI MOHAMMADIAN

Rational certificates of positivity on compact semialgebraic sets

VICTORIA POWERS

Quiver grassmannians, quiver varieties and the preprojective algebra

Alistair Savage and Peter Tingley

Nonautonomous second order Hamiltonian systems

\section{MARTIN SCHECHTER}

Generic fundamental polygons for Fuchsian groups

AKIRA USHIJIMA

Stability of the Kähler-Ricci flow in the space of Kähler metrics

KAI ZHENG

The second variation of the Ricci expander entropy 